SLAC-PUB-7309

hep-ph/9609460

September, 1996

\title{
Electron-Positron Annihilation into Four Jets at Next-to-Leading Order in $\alpha_{s}$ t
}

\author{
Adrian Signer and Lance Dixon \\ Stanford Linear Accelerator Center \\ Stanford University \\ Stanford, CA 94309
}

\begin{abstract}
We calculate the rate for $e^{+} e^{-}$annihilation into four jets at next-to-leading order in perturbative QCD, but omitting terms that are suppressed by one or more powers of $1 / N_{c}^{2}$, where $N_{c}$ is the number of colors. The $\mathcal{O}\left(\alpha_{s}^{3}\right)$ corrections depend strongly on the jet resolution parameter $y_{\text {cut }}$ and on the clustering and recombination schemes, and they substantially improve the agreement between theory and data.
\end{abstract}

Submitted to Physical Review Letters

${ }^{1}$ Research supported by the Department of Energy under grant DE-AC03-76SF00515, and by the Swiss National Science Foundation 
Jets have proven to be an extremely useful way to describe the production of hadrons in $e^{+} e^{-}$annihilation and in hadronic collisions containing large transverse momenta. Since jets can be given an infrared-safe definition [1], their properties can be calculated in perturbative quantum chromodynamics (QCD), order-by-order in the strong coupling $\alpha_{s}$. Electronpositron annihilation provides the cleanest experimental situation for studying jet properties, and large data samples from the $Z^{0}$ pole are available. On the theoretical side, there are leading order predictions for production of up to five jets [2, 3, 4, 5, 6], but to improve the precision next-to-leading-order QCD corrections are required. The $\mathcal{O}\left(\alpha_{s}^{2}\right)$ matrix elements for three-jet production and other infrared-safe quantities have been known for some time [4, 5], and numerical programs implementing these corrections [7] have been widely used to extract a precise value of $\alpha_{s}$ from hadronic event shapes [8].

Four-jet final states provide certain tests of QCD to which three-jet states are insensitive [9]. For example, the non-abelian three-gluon vertex appears at leading order in four jet events; the same is true for the production of hypothetical, light, colored but electrically neutral particles (such as light gluinos). In addition, four jet events produced directly in annihilation form a significant experimental background to the reaction $e^{+} e^{-} \rightarrow W^{+} W^{-}$ when each $W$ decays to a pair of jets, particularly when the center-of-mass energy is not far above the $W$-pair threshold, as is the case at LEP2. In this letter, we report the nextto-leading-order $\left(\mathcal{O}\left(\alpha_{s}^{3}\right)\right)$ QCD predictions for $e^{+} e^{-}$annihilation into four jets, in a large- $N_{c}$ approximation to be described shortly. The results rely heavily on the one-loop virtual matrix elements for four massless final state partons, $e^{+} e^{-} \rightarrow q \bar{q} g g$ and $e^{+} e^{-} \rightarrow q \bar{q} q^{\prime} \bar{q}^{\prime}$ [10] (for an independent calculation of $e^{+} e^{-} \rightarrow q \bar{q} q^{\prime} \bar{q}^{\prime}$, see ref. [11]), as well as the tree-level matrix elements for five final state partons, $e^{+} e^{-} \rightarrow q \bar{q} g g g$ and $e^{+} e^{-} \rightarrow q \bar{q} q^{\prime} \bar{q}^{\prime} g$ [6]. Here we give results only for the overall four-jet event rate; but the same numerical program may be used to calculate various angular distributions [12] which may test QCD more stringently.

Our main approximation to the full $\mathcal{O}\left(\alpha_{s}^{3}\right)$ QCD results for massless quarks consists of omitting terms that are suppressed by one or more powers of $1 / N_{c}^{2}$, where $N_{c}$ is the number of colors in a general $S U\left(N_{c}\right)$ gauge theory, and $N_{c}=3$ for QCD. Thus, extracting an overall factor of $\left(N_{c}^{2}-1\right)$ common to all multi-jet predictions, we write the one-loop correction to the four-jet cross-section as

$$
\sigma_{4-\text { jet }}^{1-\text { loop }}=N_{c}^{2}\left(N_{c}^{2}-1\right)\left[\sigma_{4}^{(a)}+\left(N_{f} / N_{c}\right) \sigma_{4}^{(b)}+\left(N_{f} / N_{c}\right)^{2} \sigma_{4}^{(c)}+\mathcal{O}\left(1 / N_{c}^{2}\right)+\mathcal{O}\left(N_{f} / N_{c}^{3}\right)\right]
$$

and we calculate $\sigma_{4}^{(a, b, c)}$. This is not precisely the $1 / N_{c}$ approximation of 't Hooft 13, because we keep terms that are only suppressed by $N_{f} / N_{c}$, where $N_{f}$ is the number of light fermions, $N_{f}=5$ at the $Z^{0}$ pole. The ratio $N_{f} / N_{c}$ is not small, and the $N_{f} / N_{c}$ corrections are numerically important. To assess whether the omitted $1 / N_{c}^{2}$ corrections can be expected to be small, we have evaluated the $\mathcal{O}\left(\alpha_{s}^{2}\right)$ three-jet results in the same approximation, but where we also know the full result. The size of the neglected terms varies with the precise jet definition, but it is generally about $10 \%$ of the full $\mathcal{O}\left(\alpha_{s}^{2}\right)$ correction. It is also known that the $1 / N_{c}^{2}$ terms contribute less than $6 \%$ to the $\mathcal{O}\left(\alpha_{s}^{3}\right)$ term in the expansion of the total cross-section for $e^{+} e^{-}$to hadrons [14]. In future work the $1 / N_{c}^{2}$ corrections should be available. In any case, it makes sense to break up the numerical evaluation in this way. The $1 / N_{c}^{2}$ corrections are significantly more complicated than the leading terms and therefore take longer to evaluate numerically, yet they are parametrically suppressed. Hence one can 


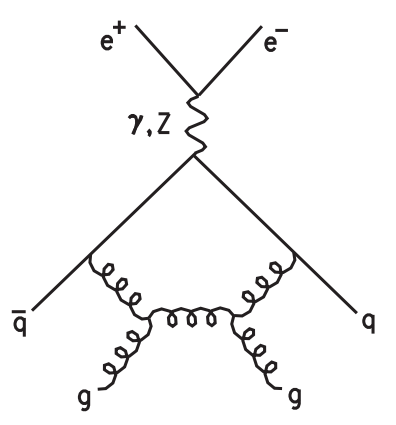

(a)

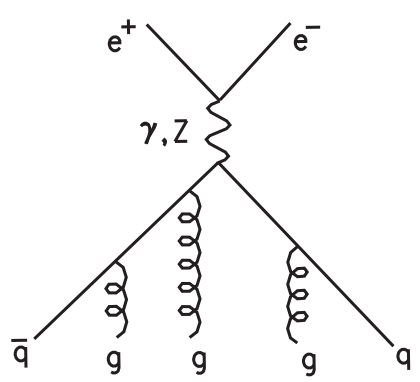

(c)

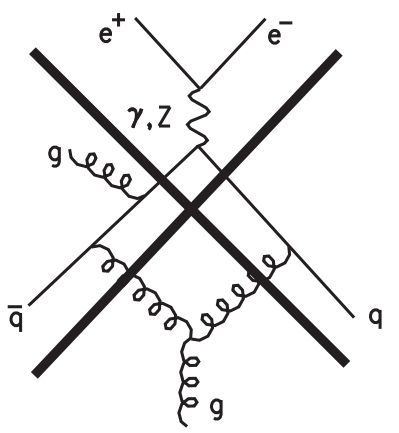

(b)

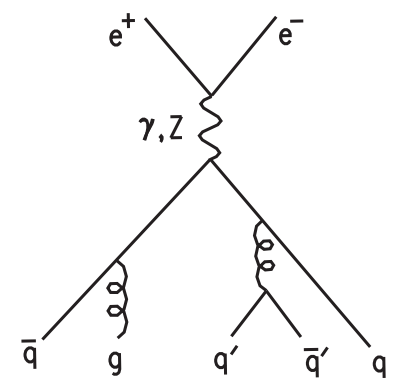

(d)

Figure 1: (a) Example of a leading-in- $N_{c}$ one-loop diagram for $e^{+} e^{-} \rightarrow q \bar{q} g g$. (b) A subleading one-loop diagram (omitted). (c) Sample tree diagram for $e^{+} e^{-} \rightarrow q \bar{q} g g g$. (d) Sample tree diagram for $e^{+} e^{-} \rightarrow q \bar{q} q^{\prime} \bar{q}^{\prime} g$.

save computer time without sacrificing overall accuracy if one separately evaluates the $1 / N_{c}^{2}$ corrections, using fewer points in their Monte Carlo integration than one uses for the leading terms. We kept all $1 / N_{c}^{2}$-suppressed terms in the tree-level $\left(\mathcal{O}\left(\alpha_{s}^{2}\right)\right)$ cross-section, except for the four-quark terms coming from Pauli-exchange (the "E" terms of ref. 画), which are numerically tiny.

We omit two other classes of $\mathcal{O}\left(\alpha_{s}^{3}\right)$ contributions:

1. Contributions proportional to the axial coupling $a_{f}$ of the $Z^{0}$ to quarks. Analogous terms have traditionally been omitted from $\mathcal{O}\left(\alpha_{s}^{2}\right)$ programs, as they cancel precisely between upand down-type quarks in the final state (for zero quark mass), and their contribution to the three-jet rate is at the percent level [15].

2. Contributions proportional to $\left(\sum_{f} v_{f}\right)^{2}$, where $v_{f}$ is the quark vector coupling. These "light-by-glue scattering" terms do not appear at $\mathcal{O}\left(\alpha_{s}^{2}\right)$ at all, have a partial cancellation from the sum over quark flavors, and contribute less than $1 \%$ to the $\mathcal{O}\left(\alpha_{s}^{3}\right)$ term in the total cross-section [14.

The $1 / N_{c}^{2}$ expansion is facilitated by using a color-ordered framework for both the oneloop and tree matrix elements [16]. The amplitudes are decomposed into kinematical quantities - partial amplitudes - multiplying particular strings (and traces) of the fundamental $S U\left(N_{c}\right)$ generator matrices $\left(T^{a}\right)_{i}{ }^{\overline{ }}$. In the expression for the cross-section, i.e. the squared amplitude, summed over all color indices, it is easy to identify the partial amplitudes that 
accompany the highest powers of $N_{c}$. They are given by the sum of color-ordered Feynman diagrams, where the cyclic ordering of the external quark and gluon legs is fixed, and where the gluons are on the opposite side of the Feynman diagram from the $e^{+} e^{-}$pair. For example, Fig. 11a shows a diagram contributing to a leading-color partial one-loop amplitude for $e^{+} e^{-} \rightarrow q \bar{q} g g$, while Fig. 1b (crossed out) shows a diagram that only contributes to subleading-color partial amplitudes. Fig. 1c and Fig. 1 $\mathrm{d}$ are sample diagrams for the five-parton tree amplitudes.

As usual, the real and virtual contributions to the cross-section are separately divergent; only the sum of the two yields a meaningful finite result. In dimensional regularization with $D=4-2 \epsilon$, the singularities of the virtual part manifest themselves as poles in $\epsilon$ in the one-loop amplitudes, whereas the real singularities are obtained upon phase-space integration of the squared tree amplitudes. In order to combine these two contributions, we use a general version of the subtraction method [4]. In fact, the program used in this letter is a straightforward implementation of the method developed in ref. [17]. We refer the reader to this article for more details. Here we only mention that no approximation of the matrix elements has to be made, and that we checked the independence of the results on the arbitrary parameters $\delta$ and $\xi_{\text {cut }}$ which have to be introduced in intermediate steps.

We now present the results for the four-jet fraction $R_{4} \equiv \sigma_{4-\text { jet }} / \sigma_{\text {tot }}$ at next-to-leading order in $\alpha_{s}$. We consider four different jet algorithms, the JADE [18], E0, Durham [19] and Geneva [20] schemes.] All four are iterative clustering algorithms: they begin with a set of final-state particles (partons in the QCD calculation) and cluster the pair $\{i, j\}$ with the smallest value of a dimensionless measure $y_{i j}$ into a single "proto-jet". The procedure is repeated until all the $y_{i j}$ exceed the value of the jet resolution parameter $y_{\text {cut }}$, at which point the proto-jets are declared to be jets. The schemes differ in the measure $y_{i j}$ used and/or in the rule for recombining two clustered momenta. The same value of $y_{\text {cut }}$ in different schemes may sample different momentum scales. The definitions of the various schemes are collected in ref. 20]. The $y_{\text {cut }}$ dependence of the results is shown in Fig. 2. In each plot, the solid (dashed) line represents the one-loop (tree-level) prediction. The renormalization scale $\mu$ has been chosen to be the center-of-mass energy $\sqrt{s}$, and we have set $N_{f}=5$ and $\alpha_{s}=0.118$ [21]. The statistical error of the Monte Carlo integration is of the order of $3 \%$. Of course, this error can be reduced further through higher statistics, but as long as the subleading-in- $N_{c}$ terms are not included, there is little point in doing so. These curves are compared to preliminary SLD data points [22] which have been corrected for detector effects and hadronization, and to available LEP1 data [23], which have not been corrected for hadronization.

The next-to-leading order results in the JADE algorithm in Fig. 2a actually agree "too well" with the data, given that the one-loop corrections are of order $100 \%$ (and that the JADE data are uncorrected for hadronization). The results in the E0 and Durham schemes (Fig. 2b,c) are more what one might expect from such large corrections: agreement to within 20 or $30 \%$. The Geneva scheme (Fig. $2 \mathrm{~d}$ ) behaves quite differently. It is the only scheme we considered where the leading-order results give a reasonable description of the data for large values of $y_{\text {cut }}$, although the shape of the prediction is not quite correct, especially at small $y_{\text {cut }}$. Here the inclusion of the one-loop correction leads to quite good agreement between

${ }^{1}$ Lacking a scheme to name after our fair city, we have dubbed our numerical program Matrix Elements for Next-to-Leading Order PARton Calculations (MENLO_PARC). 

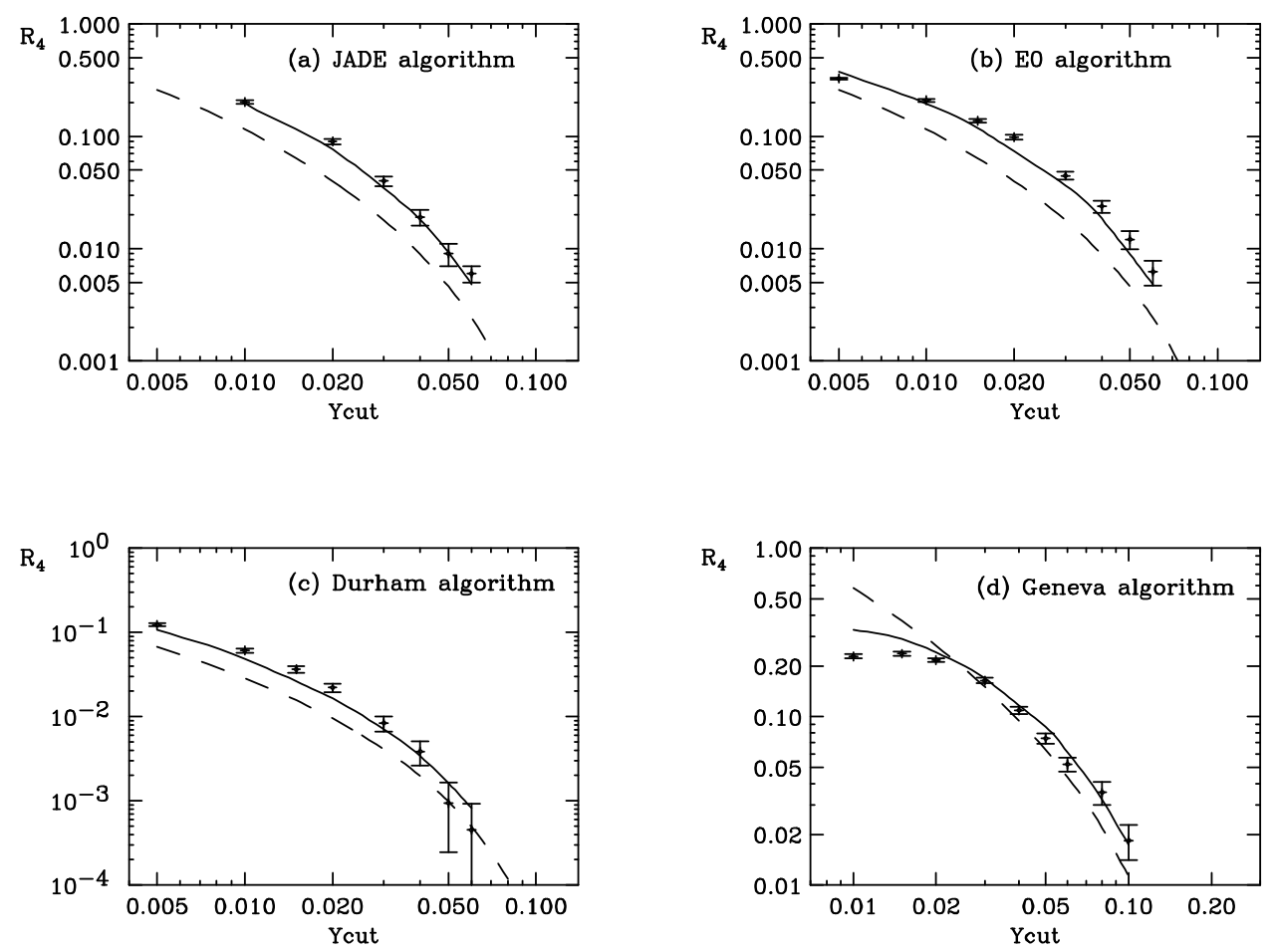

Figure 2: The four-jet fraction $R_{4}$ in $e^{+} e^{-}$annihilation, as a function of $y_{\text {cut }}$. Solid (dashed) lines represent the one-loop (tree-level) predictions in the (a) JADE, (b) E0, (c) Durham, and (d) Geneva algorithms, for $\mu=\sqrt{s}$ and $\alpha_{s}=0.118$. The data points in (a) are from DELPHI 23] (uncorrected for hadronization), while (b), (c) and (d) contain preliminary SLD data [22] (corrected for hadronization).
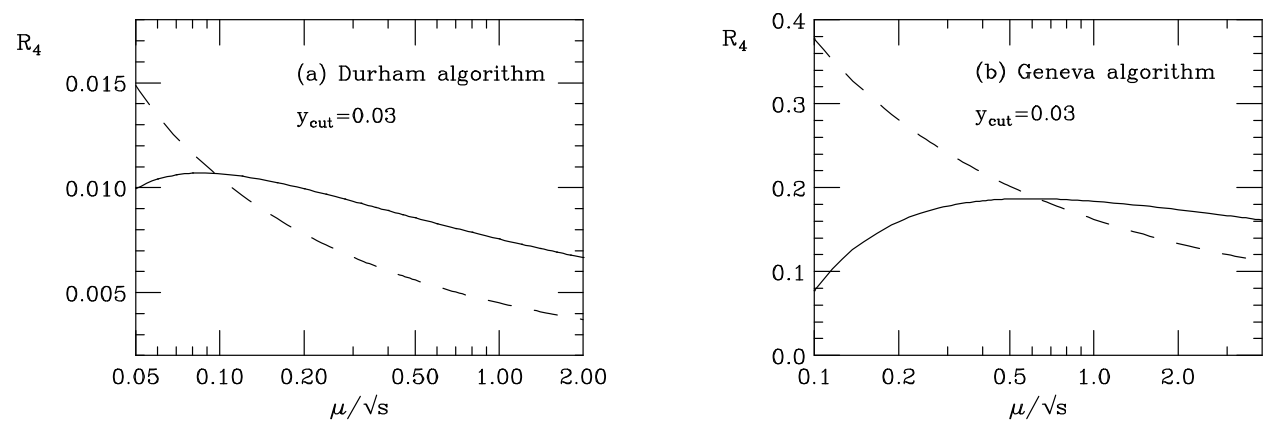

Figure 3: Solid (dashed) lines show the dependence of $R_{4}$ on the renormalization scale $\mu$ for the one-loop (tree-level) predictions in the (a) Durham, and (b) Geneva algorithms, for $\alpha_{s}=0.118$ and $y_{\text {cut }}=0.03$. 
data and theory for $y_{\text {cut }}>0.02$. However, for $y_{\text {cut }}<0.02$, the one-loop virtual corrections become very large and negative (they are dominated by the $N_{f} / N_{c}$ terms in this region), but not enough to match the strong suppression and turnover seen in the four-jet data. In any scheme, as $y_{\text {cut }}$ decreases the four-jet fraction rises quickly, but eventually it has to turn over; this phenomenon just happens at a larger value of $y_{\text {cut }}$ in the Geneva scheme than in the other schemes. We have also compared the one-loop prediction at $\sqrt{s}=35 \mathrm{GeV}$ to data from PETRA [24]. The agreement is again improved with respect to the leading-order result, but it is not quite as good as at the $Z^{0}$ pole, perhaps because hadronization effects are more important at lower energy.

The rapid fall-off of the four-jet fraction at large $y_{\text {cut }}$ means that there is little data available (at present) with which to compare our predictions for $y_{\text {cut }}>0.07$. On the other hand, for small $y_{\text {cut }}$ the QCD expansion parameter is really $\alpha_{s} \ln ^{2} y_{\text {cut }}$, and it would be advantageous to resum these large logarithms. This is possible at leading and next-toleading logarithmic order in the Durham scheme [19]. To further improve the Durhamscheme prediction, our fixed-order results could be matched [25] to the resummed results.

Observable quantities calculated in QCD should be independent of the arbitrary renormalization scale $\mu$. However, the perturbative expansion is invariably truncated at a finite order, leading to a residual dependence of the result on $\mu$. The tree-level $\mu$ dependence is much stronger for the four-jet rate than for the three-jet rate, because the former is pro-

portional to $\alpha_{s}^{2}$ instead of $\alpha_{s}$. As expected, this strong renormalization-scale dependence is reduced by the inclusion of the next-to-leading order contribution. Fig. 3 plots the $\mu$ dependence of $R_{4}$ at tree-level and at one-loop for the Durham and Geneva schemes, at $y_{\text {cut }}=0.03$. We should mention that in order to get a consistent picture, for these plots alone we omitted the corresponding $1 / N_{c}^{2}$ corrections from the tree-level term as well. On the other hand, their inclusion only slightly affects the $\mu$-dependence.

In this letter we have presented first results on the production of four jets in electronpositron annihilation at next-to-leading order in $\alpha_{s}$. These results were obtained with a numerical program which implements the subtraction method for combining real and virtual singularities as described in ref. [17]. The key ingredients for the four-jet calculation are the one-loop virtual matrix elements for $e^{+} e^{-} \rightarrow q \bar{q} g g$ and $e^{+} e^{-} \rightarrow q \bar{q} q^{\prime} \bar{q}^{\prime}$ [10, as well as the treelevel matrix elements for $e^{+} e^{-} \rightarrow q \bar{q} g g g$ and $e^{+} e^{-} \rightarrow q \bar{q} q^{\prime} \bar{q}^{\prime} g$ [ [6]. We computed the four-jet rates for the JADE, E0, Durham and Geneva schemes, neglecting terms that are suppressed by $1 / N_{c}^{2}$. Generally, the corrections are large and improve the agreement between theory and experiment considerably. The same program can be used for the computation of an arbitrary four-jet distribution at next-to-leading order. In future work we shall report on the computation of various angular distributions, including also in the program the subleadingin-color terms. These calculations may help in testing the triple gluon vertex and in the more general search for new physics in the strong interaction sector.

\section{Acknowledgement}

We thank Zvi Bern, Phil Burrows, David Kosower and Zoltan Kunszt for valuable conversations. 


\section{References}

[1] G. Sterman and S. Weinberg, Phys. Rev. Lett. 39:1436 (1977).

[2] J. Ellis, M.K. Gaillard and G.G. Ross, Nucl. Phys. B111:253 (1976), B130:516(E) (1977).

[3] A. Ali et al., Phys. Lett. B82:285 (1979); Nucl. Phys. B167:454 (1980).

[4] R.K. Ellis, D.A. Ross and A.E. Terrano, Phys. Rev. Lett. 45:1226 (1980); Nucl. Phys. B178:421 (1981).

[5] K. Fabricius, I. Schmitt, G. Kramer and G. Schierholz, Phys. Lett. B97:431 (1980); Z. Phys. C11:315 (1981).

[6] K. Hagiwara and D. Zeppenfeld, Nucl. Phys. B313:560 (1989);

F.A. Berends, W.T. Giele and H. Kuijf, Nucl. Phys. B321:39 (1989);

N.K. Falk, D. Graudenz and G. Kramer, Nucl. Phys. B328:317 (1989).

[7] Z. Kunszt and P. Nason, in Z Physics at LEP1, CERN Yellow Report 89-08;

G. Kramer and B. Lampe, Z. Phys. C34:497 (1987), C42:504(E) (1989); Fortschr. Phys. 37:161 (1989);

W.T. Giele and E.W.N. Glover, Phys. Rev. D46:1980 (1992);

S. Catani and M.H. Seymour, Phys. Lett. B378:287 (1996).

[8] OPAL Collab., P.D. Acton et al., Z. Phys. C55:1 (1992);

ALEPH Collab., D. Decamp et al., Phys. Lett. B284:163 (1992);

L3 Collab., O. Adriani et al., Phys. Lett. B284:471 (1992);

DELPHI Collab., P. Abreu et al., Z. Phys. C59:21 (1993);

SLD Collab., K. Abe et al., Phys. Rev. D51:962 (1995).

[9] S. Bethke, A. Ricker, P.M. Zerwas, Z. Phys. C49:59 (1991);

L3 Collab., B. Adeva et al., Phys. Lett. B248:227 (1990);

DELPHI Collab., P. Abreu et al., Z. Phys. C59:357 (1993);

OPAL Collab., R. Akers et al., Z. Phys. C65:367 (1995).

[10] Z. Bern, L. Dixon and D.A. Kosower, preprint hep-ph/9606378, and in preparation;

Z. Bern, L. Dixon, D.A. Kosower and S. Weinzierl, preprint hep-ph/9610370.

[11] E.W.N. Glover and D.J. Miller, preprint DTP-96-66, hep-ph/9609474.

[12] J.G. Körner, G. Schierholz and J. Willrodt, Nucl. Phys. B185:365 (1981);

O. Nachtmann and A. Reiter, Z. Phys. C16:45 (1982);

M. Bengtsson and P.M. Zerwas, Phys. Lett. B208:306 (1988);

M. Bengtsson, Z. Phys. C42:75 (1989).

[13] G. 't Hooft, Nucl. Phys. B72:461 (1974); Nucl. Phys. B75:461 (1974).

[14] S.G. Gorishny, A.L. Kataev and S.A. Larin, Phys. Lett. B259:144 (1991);

L.R. Surguladze and M.A. Samuel, Phys. Rev. Lett. 66:560 (1991), 66:2416(E) (1991). 
[15] B. Kniehl and J. H. Kühn Phys. Lett. B224:229 (1989); K. Hagiwara, T. Kuruma and Y. Yamada, Nucl. Phys. B358:80 (1991).

[16] F.A. Berends and W.T. Giele, Nucl. Phys. B294:700 (1987);

M. Mangano, S. Parke and Z. Xu, Nucl. Phys. B298:653 (1988);

M. Mangano, Nucl. Phys. B309:461 (1988);

Z. Bern and D.A. Kosower, Nucl. Phys. B362:389 (1991);

Z. Bern, L. Dixon and D.A. Kosower, Nucl. Phys. B425:217 (1994); Nucl. Phys. B437:259 (1995).

[17] S. Frixione, Z. Kunszt and A. Signer, Nucl. Phys. B467:399 (1996).

[18] JADE Collab., W. Bartel et al., Z. Phys. C33:23 (1986).

[19] N. Brown and W.J. Stirling, Z. Phys. C53:629 (1992);

S. Catani, Yu.L. Dokshitser, M. Olsson, G. Turnock and B.R. Webber, Phys. Lett. B269:432 (1991).

[20] S. Bethke, Z. Kunszt, D.E. Soper and W.J. Stirling, Nucl. Phys. B370:310 (1992).

[21] Particle Data Group, R.M. Barnett et al., Phys. Rev. D54:1 (1996).

[22] SLD Collab., K. Abe et al., Phys. Rev. Lett. 71:2528 (1993); P. Burrows (SLD Collab.), private communication.

[23] DELPHI Collab., P. Abreu et al., Phys. Lett. B247:167 (1990).

[24] TASSO Collab., W. Braunschweig et al., Phys. Lett. B214:286 (1988);

JADE Collab., N. Magnussen et al., Z. Phys. C49:29 (1991).

[25] S. Catani, L. Trentadue, G. Turnock and B.R. Webber, Nucl. Phys. B407:3 (1993). 